\title{
Optical Properties and Luminescence Behaviour of PP/Clay Nanocomposites
}

\author{
Matthieu Ambid Non-member (Université Paul Sabatier Toulouse) \\ Gilbert Teyssedre Non-member (Université Paul Sabatier Toulouse) \\ Dominique Mary Non-member (Université Paul Sabatier Toulouse) \\ Christian Laurent Non-member (Université Paul Sabatier Toulouse) \\ Gian Carlo Montanari Non-member (University of Bologna)
}

Keywords : nanocomposite, polypropylene, clay, maleïc anhydride, fluorescence, diffusion, absorption, electroluminescence

Silicate nanocomposites might be a great source of innovation for the development of advanced electrical devices since filler introduction improves mechanical properties, thermal stability and some electrical properties. In a first part of this paper we summarize results obtained by photoluminescence and UV-visible absorption spectroscopy. The optical balance (absorption vs. diffusion) is discussed and a detailed photoluminescence emission spectra analysis is provided. Grafting maleïc anhydride (MA) on PP chains induces the formation of a broad absorption band $(250-450 \mathrm{~nm})$ and changes in the photoluminescence emission spectrum whereas clay introduction increases light diffusion. In a second part we discuss results obtained using different luminescence techniques (recombination-induced luminescence RIL-, thermoluminescence and electroluminescence) in order to characterise charge recombination mechanisms and the nature of trapping centres in such materials.

Layered silicate nanocomposites (NC) are extensively studied materials. Their main interest is the onset of new properties compared to the filler and the matrix. The matrix/filler interface appears as the driving factor in the evolution of these properties. Moreover, these materials are composite so, advanced performances are expected when compared to isolated compounds.

In the case of polypropylene-based NC, it has been reported that a few percent of functional filler can improve mechanical characteristic, permeability and flammability properties. Changes in material properties due to nanostructuration have been recently reviewed.

The way to disperse silicate layer in a Polypropylene (PP) matrix is quite complex since the matrix must be rendered polar to enhance PP chains entrance into clay galleries. The clay is also modified in terms of interlayer distance and organophilic capacity by exchange of intergallery sodium cations by protonated octadecylamine (ODA). This complex synthesis route allows controlling interaction between the host material and nanograins (intercalation, exfoliation). Theoretically, nanocomposites can have new electrical properties controlled by interfaces which are dominant over bulk properties.

For application as electrical insulation, a $\mathrm{NC}$ must be characterized not only through microstructural observations, but also by using bulk electrical measurements, such as space charge, conductivity, dielectric spectroscopy and optical properties.

It has been shown that the space charge threshold and space charge decay characteristics are affected by increasing filler content. This has been attributed to a change in trap distribution. We have used a set of luminescence techniques in order to probe luminescent centres in this kind of materials and to identify chemical species that are electrically active, being involved in charge trapping and in recombination processes.

We focus our attention on optical properties (absorption, diffusion) and luminescence properties (photoluminescence, recombination-induced luminescence, thermoluminescence and electroluminescence), with the aim of inferring characterization of NC polymers, with particular regard to trapping, luminescence centres and charge transport features.

We have performed a broad optical caracterisation of PP/clay $\mathrm{NC}$ with a special attention to matrix nature (tacticity, blended or not) and clay concentration. The main results can be summarized as follows:

- It appears that compatibilisation of PP chain modifies strongly photoluminescence and absorption characteristics. The origin of these modifications are due to MA compatibilisation by products or/and to PP oxidized groups.

- In NCs, the optical characteristics are those of oxidized PP. It is probably due to the reduction of antioxidant efficiency.

- Light scattering in NCs is both clay and crystallinity dependent, the latter being the most influential factor.

- RIL shows that no additional radiative recombination centres are introduced upon MA grafting or filler introduction. It seems that oxidized PP is providing the recombination centres.

- The thermoluminescence curves show an increase in the number of trapped carriers in SPP matrix based NC and a decrease has been observed in iPP matrix based NC. These observations are in agreement with space charge density measured at $30-40 \mathrm{kV} / \mathrm{mm}$ after a poling period of $10000 \mathrm{~s}$ with by the pulse electro-acoustic method.

- Filler introduction in the matrix decreases the EL threshold.

Thermostimuled current measurements will be performed soon to complete the traps characterisation.

Overall, this work show that chemical/morphological modications needed for $\mathrm{NC}$ processing are critical for optical and luminescence properties. This could also be the case for electrical properties: it is highly challenging to relate changes of electrical properties with the nanometric nature of the clay. 


\title{
Optical Properties and Luminescence Behaviour of PP/Clay Nanocomposites
}

\author{
Matthieu Ambid* \\ Non-member \\ Gilbert Teyssedre* \\ Non-member \\ Dominique Mary* \\ Non-member \\ Christian Laurent* \\ Non-member \\ Gian Carlo Montanari** Non-member
}

\begin{abstract}
Silicate nanocomposites might be a great source of innovation for the development of advanced electrical devices since filler introduction improves mechanical properties, thermal stability and some electrical properties. In a first part of this paper we summarize results obtained by photoluminescence and UV-visible absorption spectroscopy. The optical balance (absorption vs. diffusion) is discussed and a detailed photoluminescence emission spectra analysis is provided. Grafting maleïc anhydride (MA) on PP chains induces the formation of a broad absorption band (250-450nm) and changes in the photoluminescence emission spectrum whereas clay introduction increases light diffusion. In a second part we discuss results obtained using different luminescence techniques (recombination-induced luminescence - RIL-, thermoluminescence and electroluminescence) in order to characterise charge recombination mechanisms and the nature of trapping centres in such materials.
\end{abstract}

Keywords : nanocomposite, polypropylene, clay, maleïc anhydride, fluorescence, diffusion, absorption, electroluminescence

\section{Introduction}

Layered silicate nanocomposites (NC) are extensively studied materials. Their main interest is the onset of new properties compared to the filler and the matrix. The matrix/filler interface appears as the driving factor in the evolution of these properties ${ }^{(1)}$. Moreover, these materials are composite so, advanced performances are expected when compared to isolated compounds.

In the case of polypropylene-based $\mathrm{NC}$, it has been reported that a few percent of functional filler can improve mechanical characteristic $^{(2)}$, permeability ${ }^{(3)}$ and flammability properties ${ }^{(4)}$. Changes in material properties due to nanostructuration have been recently reviewed ${ }^{(5)}$.

The way to disperse silicate layer in a Polypropylene (PP) matrix is quite complex since the matrix must be rendered polar to enhance PP chains entrance into clay galleries. The clay is also modified in terms of interlayer distance and organophilic capacity by exchange of intergallery sodium cations by protonated octadecylamine (ODA). This complex synthesis route allows controlling interaction between the host material and nanograins (intercalation, exfoliation) $)^{(1)(6)}$. Theoretically, nanocomposites can have new electrical properties ${ }^{(1)}$ controlled by interfaces which are dominant over bulk properties.

For application as electrical insulation, a $\mathrm{NC}$ must be characterized not only through microstructural observations, but also by using bulk electrical measurements, such as space charge, conductivity, dielectric spectroscopy and optical properties.

It has been shown that the space charge threshold and space charge decay characteristics are affected by increasing filler content $^{(7)}$. This has been attributed to a change in trap distribution.

* Laboratoire de Génie Electrique, Université Paul Sabatier Toulouse III - CNRS, 118 route de Narbonne, 31062 Toulouse, France

** Department of Electrical Engineering, University of Bologna Viale Risorgimento 2, 40136 Bologna, Italy
We have used a set of luminescence techniques in order to probe luminescent centres in this kind of materials and to identify chemical species that are electrically active, being involved in charge trapping and in recombination processes.

We focus our attention on optical properties (absorption, diffusion) and luminescence properties (photoluminescence, recombination-induced luminescence, thermoluminescence and electroluminescence), with the aim of inferring characterization of NC polymers, with particular regard to trapping, luminescence centres and charge transport features.

\section{Experimental}

2.1 Materials Polypropylene nanocomposites were prepared from organophilic layered silicate, using synthetic fluorohectorite (Somasif ME 100 from CO-OP) modified with an alkyl ammonium cation (octadecylamine (ODA)). Isotactic polypropylene (iPP) used is referenced PP HC 01 A-B1 from Borealis. The iPP compatibilizer, provided by Clariant (Licomont AR 5040), is a PP containing 3.5 wt- $\%$ maleic anhydride (MA) grafted to the backbone of the chain of the polymer. The matrix of the investigated composite materials, referred to as iPP-20AR in the following is a blend of iPP and compatibilized iPP with a ratio of 80:20 wt.\%. Measurements were carried out on pure iPP, and on iPP-20AR with 0 to 6 wt.\% of modified fluorohectorite. For syndiotactic polypropylene (sPP) based composites, the matrix contained 0.4 wt- $\%$ of grafted MA (from Atofina) as a compatibilizer. We carried out measurements on samples with 0,6 and 20 wt. $\%$ of modified fluorohectorite. All samples were stabilized with Irganox-1010/Irgafos168 (4:1) from Ciba. Specimens were extruded at $210^{\circ} \mathrm{C}$ in film form of c.a. $400 \mu \mathrm{m}$ thickness.

2.2 Techniques All luminescence experiments were performed in the same cell, being equipped with different excitation sources (photon, cold plasma, temperature, electric 
field). The cell is equipped for both integral and spectral analysis of the light emitted by the stressed sample. Two detectors are used: a photomultiplier for integral light analysis (e.g. thermoluminescence glow curves, electroluminescence) and a CCD camera from Princeton Instrument (LN/CCD-1100-PB) coupled to a Jobin-Yvon CP200 grating monochromator for spectral analysis.

The light-tight chamber is equipped with a pumping unit. It is thermally-controlled from $-130^{\circ} \mathrm{C}$ to up to $150^{\circ} \mathrm{C}$ using a liquid nitrogen flow and a heating resistance. Experiments were performed under nitrogen or helium atmosphere from secondary vacuum $\left(10^{-4} \mathrm{mBar}\right)$ to $1200 \mathrm{mbar}$.

(1) Photoluminescence The continuous excitation is provided by a Xenon lamp (150 Watts) coupled to a Jobin-Yvon HD10UV monochomator so that the excitation wavelength is selected in the wavelength range $200-800 \mathrm{~nm}$. Samples are excited using light beam from $220 \mathrm{~nm}$ to $300 \mathrm{~nm}$ as in general the major emitting species in polyolefins are believed to be carbonyl and aromatic compound or impurities. Measurements were carried out in helium at atmospheric pressure as thermal exchange gas.

Three kinds of measurements were carried out:

- photoluminescence (PL) emission spectra at various excitation wavelengths, at room temperature, where we usually obtain essentially fluorescence emission;

- same experiment at low temperature $\left(-130^{\circ} \mathrm{C}\right)$, where phosphorescence can be probed.

- PL spectra vs. temperature (at a heating rate of $5^{\circ} \mathrm{C} / \mathrm{min}$ ) for a fixed excitation wavelength.

(2) UV-visible absorption Absorbance measurements have been performed on two different recording spectrometers. The first one is a Shimadzu UV-2100 spectrometer allowing a direct measure of the transmitted beam. It means that absorbed and scattered light contributions can not be separated. The second one is a Perkin-Elmer Lambda 900, provided with an integrating sphere. The sample is installed at the entrance of the sphere. This configuration provides the true absorbance of the material since scattered beams are collected. In both cases, the incident light wavelength varies from $250 \mathrm{~nm}$ to $600 \mathrm{~nm}$.

(3) Differential Scanning Calorimetry (DSC) The setup used is a DSC 2010 TA instrument. Samples of about $8 \mathrm{mg}$ weight have been tested with air as reference. Ramp rates were of $10^{\circ} \mathrm{C} / \mathrm{min}$. The temperature cycle was as follows: heating from $25^{\circ} \mathrm{C}$ to $195^{\circ} \mathrm{C}$, then cooling down to $25^{\circ} \mathrm{C}$ to $195 \mathrm{C}$ to $25^{\circ} \mathrm{C}$ and repeating again the two ramps.

(4) RIL and thermoluminescence Recombination-induced luminescence - RIL- $^{(8)}$ provides signatures of optically active trap centres involved in recombination processes. Short interaction time $(5 \mathrm{~s})$ of the helium plasma with the material surface was used in order to induce negligible surface modification of the tested sample. The decay of light emitted by the surface, after the discharge was switched off, was analysed in both the integral and wavelength-resolved forms. Luminescence excited through processes other than radiative recombination usually occurs a short time after the end of the excitation. In this work, reference will only be made to the emission spectra recorded in a time range where charge recombination was found to be the dominant excitation mechanism, i.e. at time $>50$ s. Experiments were undertaken at $-130^{\circ} \mathrm{C}$ in a helium gas at atmospheric pressure.

Thermoluminescence (TS) was recorded with the PM after waiting for $20 \mathrm{~min}$ in isothermal conditions once the discharge has been applied. By doing so, the isothermal luminescence decayed to a low level prior to heating. The heating rate was $5^{\circ} \mathrm{C} / \mathrm{min}$ from -130 up to $35^{\circ} \mathrm{C}$.

(5) Electroluminescence Semi-transparent gold electrodes were deposited on the sample surfaces by cold sputtering. The sample is positioned between two cylindrical polished electrodes brass. The top electrode, connected to HV, was a ring, which allowed light detection in the central part. In order to minimize edge effects and avoid discharges, all tests were performed in a high vacuum $\left(10^{-4} \mathrm{mbar}\right)$, with the electrode edges covered with silicone rubber. Moreover, a plastic cover was used to detect only light coming from the centre of the upper electrode. The EL signal was detected with the PM and the external current was measured from the lower electrode by a Keithley 617 electrometer. Measurements were performed at room temperature. The electrical stress cycle applied is a step-wise DC voltage increase from 0 to $60 \mathrm{kV} / \mathrm{mm}$ with steps in field of $2.5 \mathrm{kV} / \mathrm{mm}$ of $5 \mathrm{~min}$ in duration each. At the end of the step-wise cycle, voltage was set to 0 and the depolarization current was recorded during one hour. We have repeated this ramp several times on the same sample to check reproducibility and to investigate pre-stressing effects.

\section{Luminescence and Optical Balance}

3.1 Photoluminescence Emission spectra obtained at room temperature are shown in Fig. 1 and in the sum-up Table 1. An emission at $338 \mathrm{~nm}$ is observed in all the samples for an excitation at $220-230 \mathrm{~nm}$. This is the usual fluorescence emission reported for polypropylene ${ }^{(9)}$. Several authors attempted to isolate
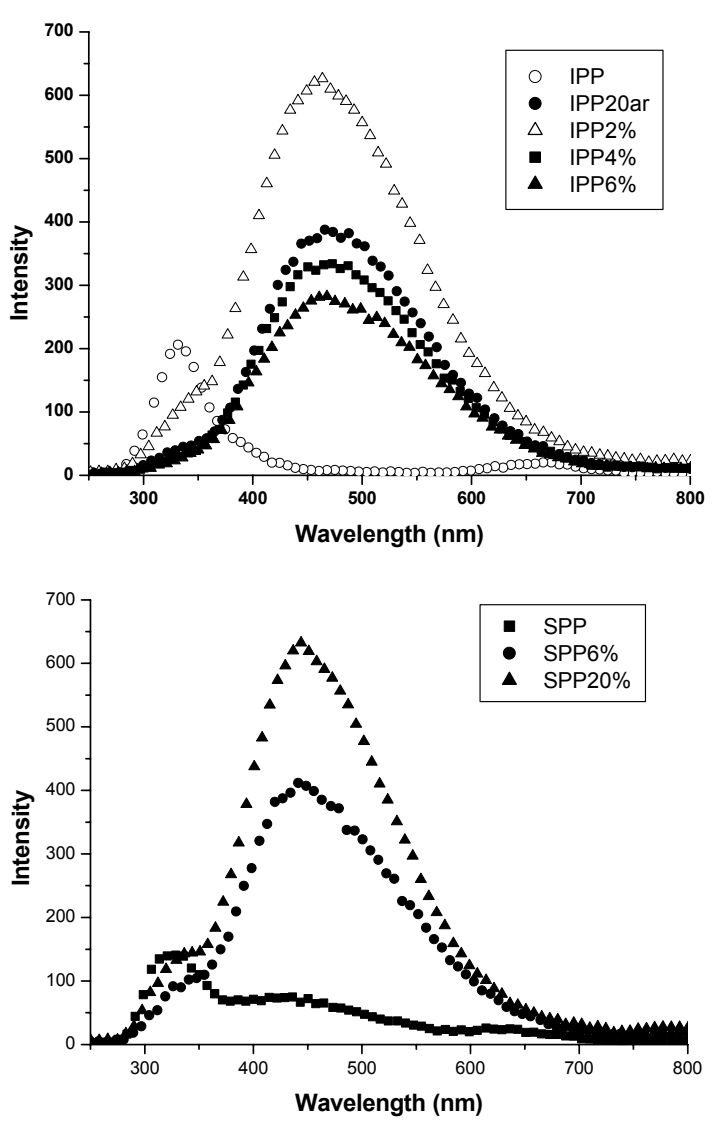

Fig. 1. Photoluminescence spectra of the various materials excited at $290 \mathrm{~nm}$ at room temperature, intensities are in arbitrary unit 
Table 1. Sum-up of photoluminescence results at $25^{\circ} \mathrm{C}\left(\lambda_{\max }\right.$ corresponds to the peak in excitation (EX) or emission (EM))

\begin{tabular}{l|l|l}
\hline Polymer & $\lambda_{\max } \mathrm{EX}(\mathrm{nm})$ & $\lambda_{\max } \mathrm{EM}(\mathrm{nm})$ \\
\hline IPP & $220-230$ & 338 \\
& 280 & 338 (weak) \\
& 250 & 375 (weak) \\
\hline IPP 2\% & $220-230$ & 237 \\
& 290 & 460 \\
\hline IPP 4\% & $220-230$ & 338 \\
& 290 & 460 \\
\hline IPP 6\% & 230 & 338 \\
& 290 & 465 \\
\hline IPP 20 AR & 230 & 338 \\
& 290 & 465 \\
\hline SPP (0.4MA) & 230 & 338 \\
& 280 & 323 (weak) \\
& $290($ lim) & 430 (weak) \\
\hline SPP 6\% & 230 & 338 \\
& 290 & 450 \\
\hline SPP 20\% & $220-230$ & 338 \\
& 290 & 450 \\
\hline
\end{tabular}

the structural element that fluoresce in this wavelength range but actually, its origin is not clear ${ }^{(9)}$. The fluorescence of iPP with excitation at $280 \mathrm{~nm}$ and emission at $336 \mathrm{~nm}$, noted as $280 \mathrm{EX} / 336 \mathrm{EM}$, would be due to enone groups. Emission in this range is therefore linked with the oxidation of the base material.

For iPP, an emission band appears at around $460 \mathrm{~nm}$ (290EX) as soon as maleic anhydride is introduced (cf. IPP20AR and NCs)). Introducing nanoparticles apparently does not change qualitatively the spectrum. As regards intensity vs. clay content, there is no clear trend in iPP-based NCs.

In $\mathrm{SPP}$ (containing $0.4 \% \mathrm{MA}$ ), an emission band is detected at $430 \mathrm{~nm}$. In sPP-based NCs, an emission band is present in the same range, but its maximum is slightly shifted towards longer wavelength (peak at $450 \mathrm{~nm}$ ). The emission intensity clearly increases with clay concentration. Note that all sPP-based samples contain the same concentration of maleic anhydride and hence the evolutions have to be explained by features linked to clay introduction.

Figure 2 shows the emission spectra of $\mathrm{SPP} 6 \%$ at $-30^{\circ} \mathrm{C}$ for two different excitation wavelengths of 230 and $280 \mathrm{~nm}$. Compared to the spectrum of the same sample at room temperature (Fig. 1), it can be observed that there are no strong differences in the spectral shape. It means that phosphorescence, if any, is also seen at room temperature.

In order to investigate the temperature dependence of the photoluminescence features, spectra were acquired in $5^{\circ} \mathrm{C}$ intervals while increasing the temperature at a rate of $5^{\circ} \mathrm{C} / \mathrm{min}$ from -130 to $30^{\circ} \mathrm{C}$. Figures 3 and 4 clearly show different behaviours for iPP and iPP loaded with $2 \%$ organoclay. At $-130^{\circ} \mathrm{C}$, the nanocomposite emits at around $470 \mathrm{~nm}$. The natural fluorescence of iPP at around $330 \mathrm{~nm}$ is always present, although much weaker. We have found similar results for iPP20AR, iPP2\% and iPP $6 \%$.

So, the introduction of maleic anhydride induces a broad emission band between 400 and $550 \mathrm{~nm}$, at any temperature. In order to provide a complete optical characterisation and to make an optical balance we have performed UV-visible absorption measurements.

3.2 UV-visible Absorption Fig. 5 shows absorbance results for iPP based samples. The strong absorption band between 250 and $375 \mathrm{~nm}$ appears when MA is grafted to the PP chains.

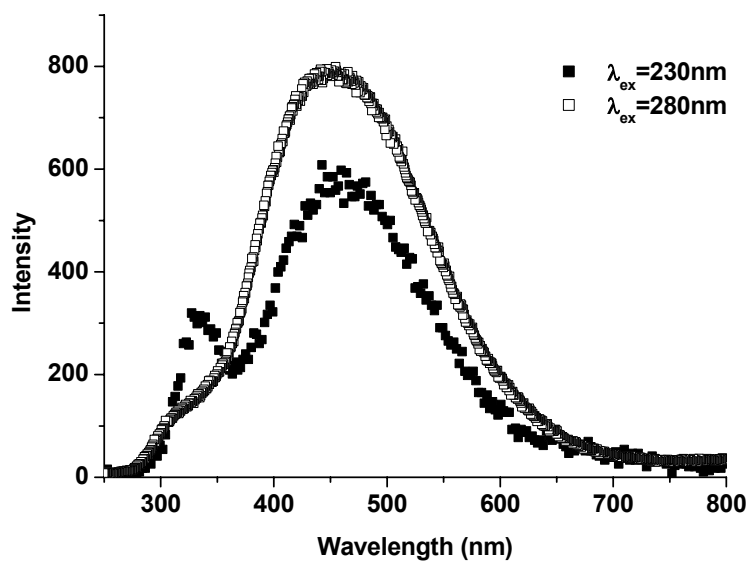

Fig. 2. Photoluminescence spectra of SPP 6\%, excited at 230 $\mathrm{nm}$ and $280 \mathrm{~nm}$, at $-130{ }^{\circ} \mathrm{C}$, intensities are in arbitrary unit. Spectra's are corrected for the spectral response of the excitation source and related optics

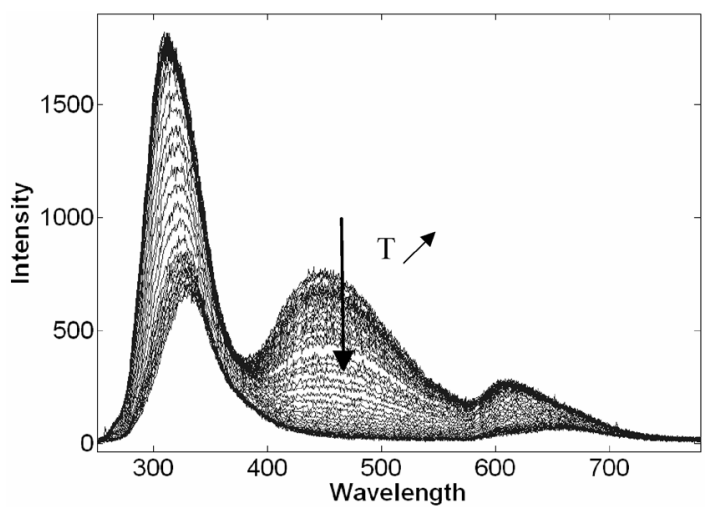

Fig. 3. Temperature dependence of photoluminescence of iPP excited at $280 \mathrm{~nm}$ (from $-130^{\circ} \mathrm{C}$ to room temperature); The band peaking in the range $620-660 \mathrm{~nm}$ is the $2^{\text {nd }}$ order of the emission between $310-330 \mathrm{~nm}$ and is therefore not due to PL. Intensities are in count per $10 \mathrm{~s}$

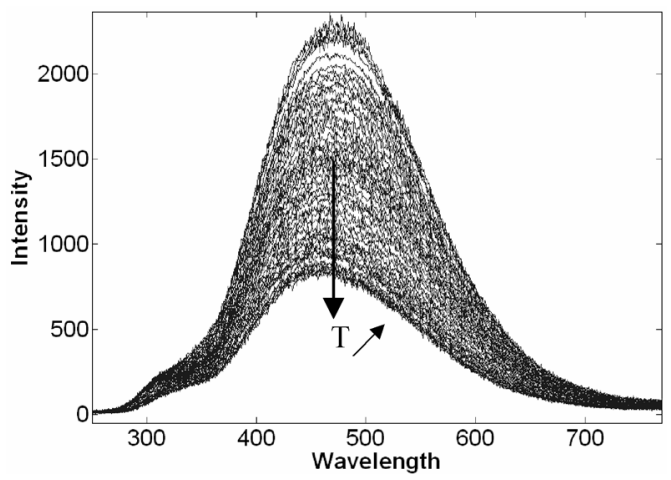

Fig. 4. Temperature dependence of photoluminescence of iPP $2 \%$ excited to $280 \mathrm{~nm}$ (from $-130^{\circ} \mathrm{C}$ to room temperature); Intensities are in count per $10 \mathrm{~s}$

However, a previous study ${ }^{(10)}$ has been reported an absorption band from 215 to $265 \mathrm{~nm}$ in MA grafted PP film. It has been attributed to the formation of PP degradation product ${ }^{(10)}$ and to phenolic antioxidants. The larger absorption band observed could be linked to the formation of new product during the compatibilization compared to ${ }^{(10)}$. It is certainly linked to 


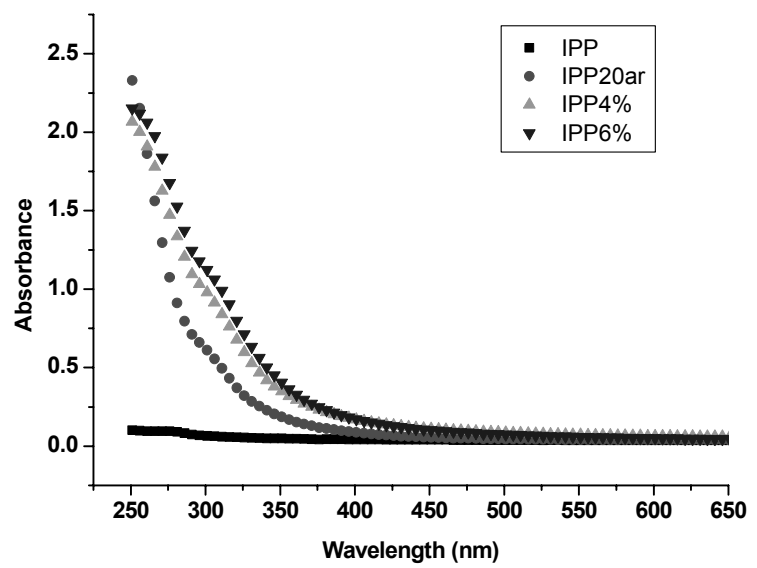

Fig. 5. UV-visible absorption for samples based on iPP matrix using an integrating sphere

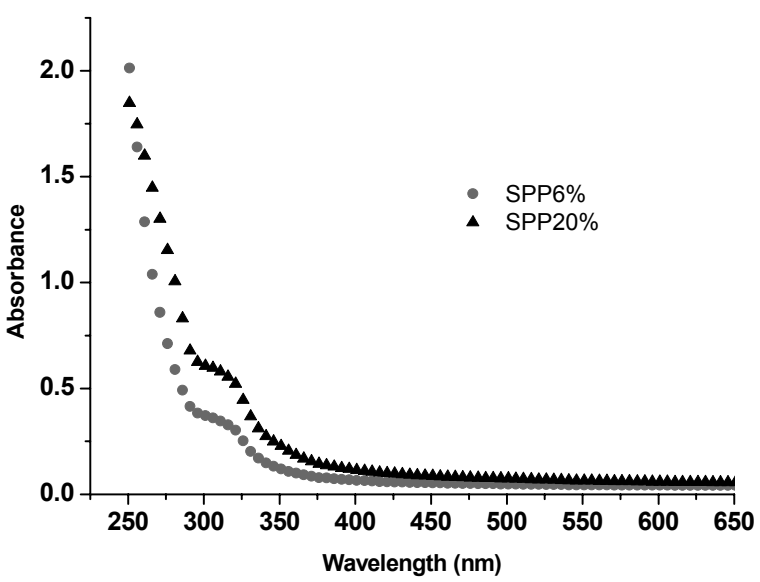

Fig. 6. UV-visible absorption for samples based on sPP matrix using an integrating sphere

compounds used during MA grafting and maybe to antioxidants. Grafting MA on the backbone of PP is done using peroxides. During this process several chemical reactions are competitive. We suppose that there is, in compatibilized PP, a reaction between $\mathrm{PP}$ and compounds used during grafting process (such as MA, peroxides) that involves formation of new chemical functions. The modification of absorbance characteristics in iPP20ar compared to PP or MA products formed during processing. The introduction of clay increases the absorption in the range $275-400 \mathrm{~nm}$ but without obvious concentration effect. This increase can be linked to the mofication of PP enhanced by the presence of the clay. Indeed according to ${ }^{(11)}$, a localisation of antioxidant close to clay layers during processing decreases antioxidant action on PP chains.

Since excited molecules can relax their energy through radiative and non-radiative transitions, the photoluminescence excitation spectrum (emission intensity vs excitation wavelength) does not map necessarily the absorption spectrum. But we can point out that compatibilization of the matrix is critical for both photoluminescence and absorbance properties. The absorption band at $290-310 \mathrm{~nm}$ corresponds to the excitation wavelength range where fluorescence at $460-465 \mathrm{~nm}$ is maximum.

Figure 6 shows UV-visible absorption spectra of sPP matrix based nanocomposites. We observe a large absorption region from 250 to $375 \mathrm{~nm}$ with a specific band at $290-320 \mathrm{~nm}$. The absorption increases with clay concentration, especially between $290 \mathrm{~nm}$ and

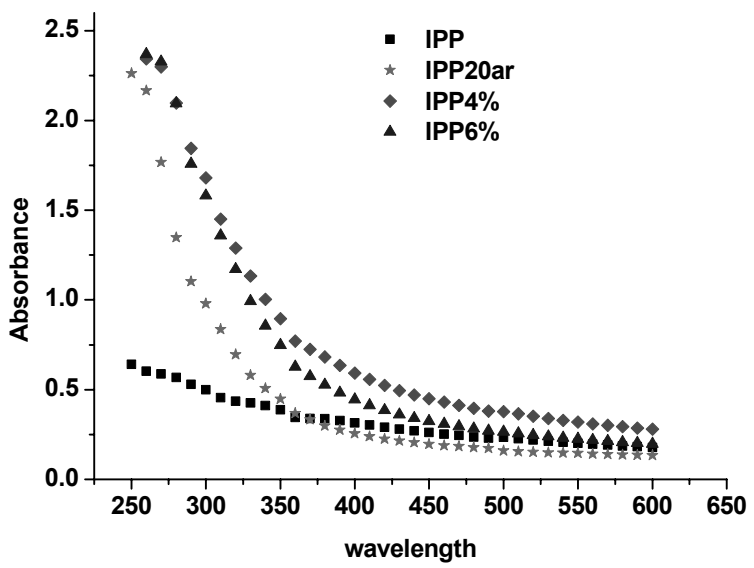

Fig. 7. UV-visible absorption for samples based on iPP matrix measured without integrating sphere

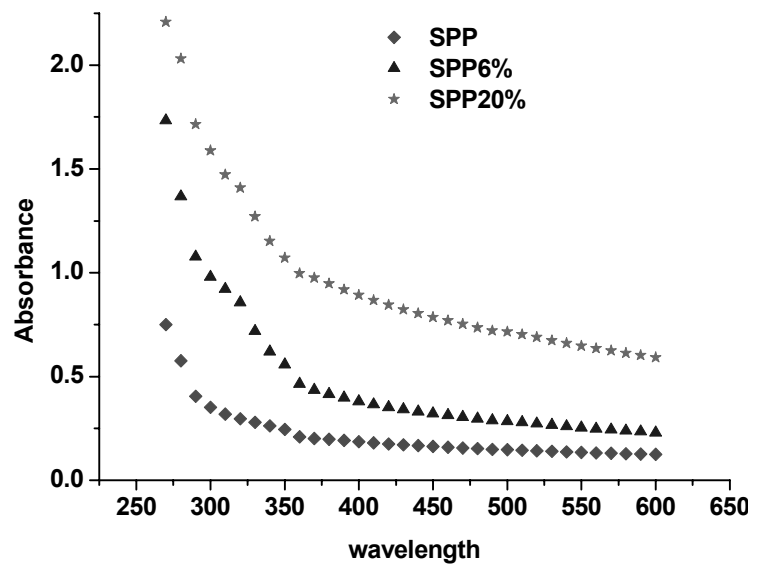

Fig. 8. UV-visible absorption for samples based on sPP matrix measured without integrating sphere

$320 \mathrm{~nm}$, but it is not directly proportional to clay concentration. This behaviour has been also observed in fluorescence where the emission intensity is fostered when the clay concentration increases.

\subsection{Scattering Behaviour of the Matrix and Clay}

Figures 7 and 8 shows the absorbance measured with the UV-visible spectrometer without integrating sphere for iPP and sPP matrix based samples respectively. Comparing Figs. 5 and 6 (measurements with the integrating sphere) with Figs. 7 and 8, it can be noticed that "absorbances" are quantitatively comparable in the range $250-350 \mathrm{~nm}$. This is not anymore the case in the range $350-600 \mathrm{~nm}$ where the apparent absorbance measured without integrating sphere is much higher. This is in fact due to both absorption (weak) of the materials and light diffusion since there is no integrating sphere. Several kinds of diffusion centres can be present in our materials: spherulites ( $\mathrm{PP}$ is a semicrystalline polymer), clay cluster, clay sheet, rough surfaces...

In order to characterise in a simple way this scattered light we calculated the difference between transmission coefficients measured with and without integrating sphere. We called it diffusion coefficient; it represents the percentage of the incident light intensity that has been scattered, since we neglected the reflected light by the sample. The corresponding spectrum is presented on Fig. 9. iPP has a diffusion coefficient that decreases linearly with the wavelength. The diffusion coefficient of compatibilized samples increases from 250 to $400 \mathrm{~nm}$ (350 nm for 


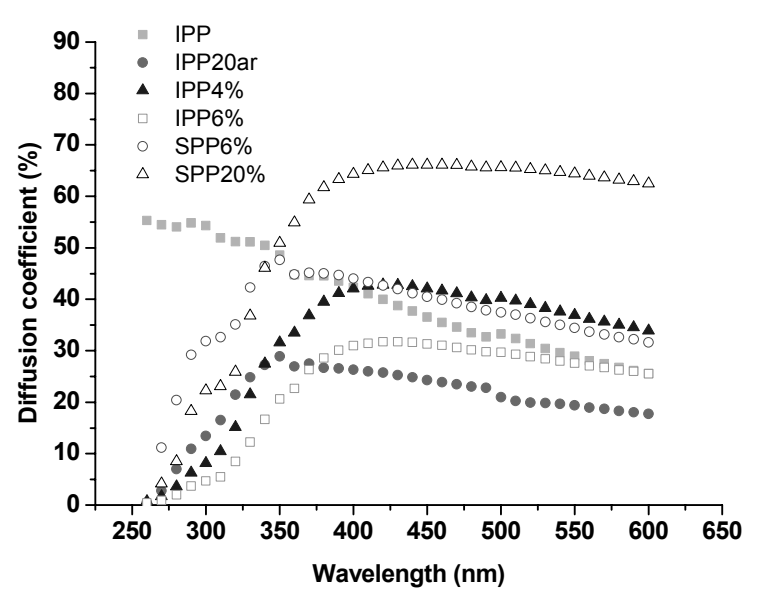

Fig. 9. Diffusion coefficient calculated from the difference between transmission coefficients measured with and without integrating sphere

Table 2. Crystallinity estimation from DSC data from the first heating scan; All data are corrected for the weight of clay

\begin{tabular}{lcc}
\hline Sample & Weight (mg) & Xc (\%) \\
iPP & 8 & 43 \\
iPP20AR & 6.2 & 46 \\
iPP4\% & 8.6 & 43 \\
iPP6\% & 9 & 37 \\
sPP & 8.7 & 24 \\
sPP6\% & 8.2 & 16 \\
sPP20\% & 9.3 & 28 \\
\hline
\end{tabular}

iPP20ar) then decreases from 400 to $600 \mathrm{~nm}$.

The range [250-400nm] can be called diffusion coefficient extinction zone since there is no diffusion in this wavelength range. The light is absorbed by MA or by products formed during the compatibization process as discussed earlier.

The longer wavelength range, [400-600nm], really reflects diffusion processes and we need to consider it in terms of structural features of the materials, through crystallinity, spherulite size, clay concentration, presence or not of clay cluster (clay aggregate size), surface roughness. We can discard the later since all samples have the same surface state. We note that the slope of the diffusion coefficient as a function of wavelength is modified by the compatibilization process. It is less wavelength dependant when MA is grafted. It has been shown that grafting MA on the PP chains produces smaller average spherulite diameter $(50 \%$ smaller than in pure PP) ${ }^{(12)}$. The difference observed is probably linked to this morpholology modification since light scattering occurs in a semicrystalline polymer mostly because of the difference on refractive indexes between crystallites and amorphous phase ${ }^{(13)}$. The influence of MA on the refractive index difference can also be an important factor on the scattering behaviour.

To estimate the crystallinity of our samples we must assume that clay plays just a role as nucleation agent and does not affect the heat of fusion of polypropylene. For iPP, the literature gives $209 \mathrm{~J} / \mathrm{g}$ as heat of fusion ${ }^{(14)}$. For sPP, the degree of crystallinity was calculated (cf. Table 2) by assuming the heat of fusion of perfectly crystalline syndiotactic polypropylene to be $166 \mathrm{~J} / \mathrm{g}^{(15)}$. As sPP is modified by MA, this is somewhat like a new material, and so this value may be wrong. This also holds for compatibilized iPP. So, in both cases, we will perform qualitative conclusion on crystallinity.

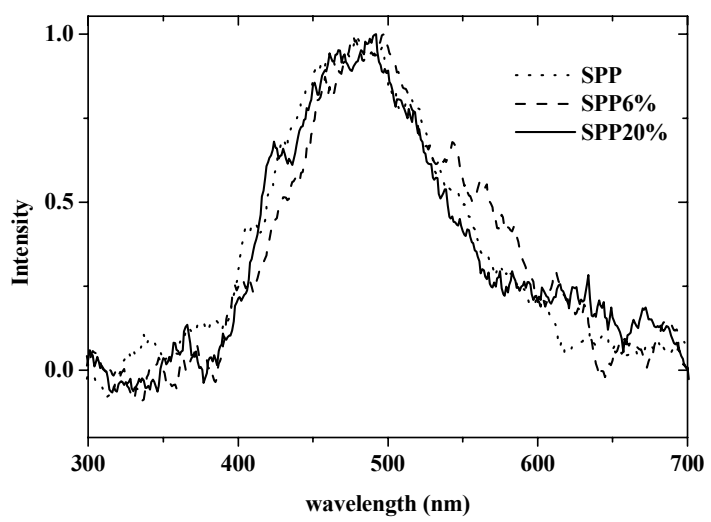

Fig. 10. RIL spectra for samples based on SPP matrix. Insenties are normalized to spectra's maximum

During NC processing, modifications of spherulite size, growing rate and density have been observed ${ }^{(16)}$. iPP4\% is less crystalline than iPP20ar (cf. Table 2) and it diffuses more. So we can suppose that the clay has an important role in diffusion results. It is certainly the case since there are different levels of clay particle (isolated platelet, tactoïd) present in the $\mathrm{NC}^{(17)}$. The more numerous particles are nanometric scale; hence, Rayleigh approximation $^{(13)}(k a<<1$ with $k$ as wave vector and $a$ as particule size) is verified. These small particles enhance the scattering effect of the matrix.

For NCs based on iPP matrix, in the range 400-600nm the diffusion coefficient is shifted to lower value when clay concentration increases. We can make a correlation between theses values and the crystallinity (cf. Table $2-43 \%$ vs. $37 \%$ for $4 \%$ and $6 \%$ clay). For sPP matrix based NC it is also possible to make this correlation since the diffusion coefficient increases with the crystallinity ( $16 \%$ vs. $28 \%$ for $6 \%$ and $20 \%$ clay).

So we can conclude that the scattering light in a NC is controlled by the clay particles and by the crystalliny rate.

\section{RIL Measurements}

Figure 10 shows the different RIL spectra obtained for sPP samples. We have found similar results for iPP-based samples (not shown here). The signal recorded was very low, leading to somewhat noisy spectra. Figure 10 shows that emission from all samples peaks at about $490 \mathrm{~nm}$ and that there is no significant influence of filler content. From these results, and those of the iPP-series, it can be concluded that there is no additional optical recombination centre after compatibilization or clay addition. The obtained spectra appear typical of the signature of oxidized $\mathrm{PP}^{(18)}$.

Since RIL spectra do not change much when going to pure iPP, to compatibilized iPP or SPP and to NCs, what seems actually observed is charge recombination from oxidized PP groups localized at the surface of the films. This appears as a very different trend from that obtained in photoluminescence, where compatibilization of iPP and filler introduction into sPP had a significant effect.

\section{Thermoluminescence}

For RIL measurement purpose, charge deposition is achieved by plasma interaction at low temperature $\left(-130^{\circ} \mathrm{C}\right)$ where thermal detrapping is weak and chain configurations are frozen. When the temperature is increased, electrons/holes are released from traps by thermal detrapping, tunnelling or traps disappearance by chain 


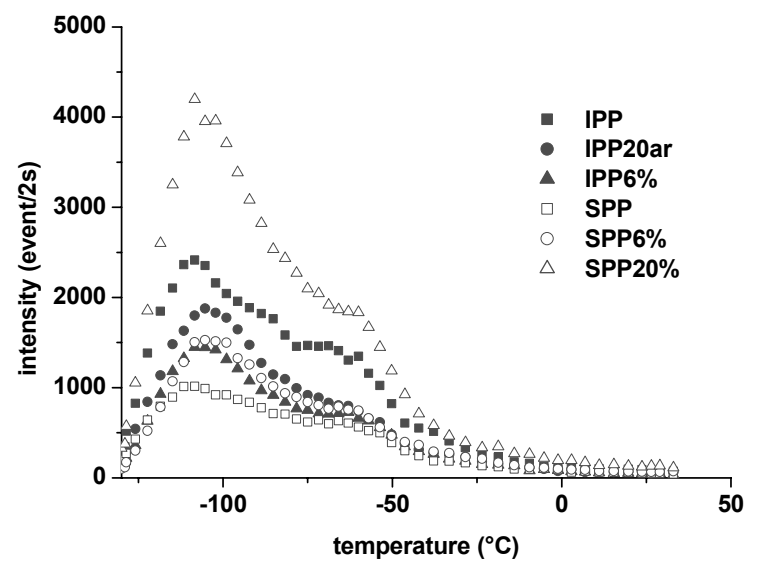

Fig. 11. TL intensity vs. temperature recorded during heating up at $5^{\circ} \mathrm{C} / \mathrm{min}$ after isotherm RIL measurements

reconfiguration. Then electrons/hole pairs can recombine on luminescent recombination centers ${ }^{(19)}$. Since we have not seen differences between RIL spectra (in terms of intensity and wavelength dependence) we can suppose that one kind of recombination center is involved.

Figure 11 shows the glow curve (thermoluminescence signal) $-\mathrm{TL}$ - recorded during increase of the temperature about $20 \mathrm{~min}$ after the plasma excitation switch-off (isothermal post-discharge luminescence was measured in this time interval). There is no strong difference in the shape of glow curves: two peaks are observed at about $-110^{\circ} \mathrm{C}$ and $-60^{\circ} \mathrm{C}$. This means that the phenomena have the same activation energy and therefore that the mechanism controlling the detrapping is the same. This is an important point since thermoluminescence peaks are usually related to polymer relaxations. PP has a $\gamma$ relaxation at $-80^{\circ} \mathrm{C}$ and the detrapping mechanism can be link to a change in trap depth associated with this relaxation. But it is difficult to conclude since PP $\gamma$ relaxation dependence on compatibization or on tacticity or on clay adding have not been investigated. There is no apparent difference around the $\beta$ relaxation (normally found at around $0^{\circ} \mathrm{C}$, depending on excitation frequency in dynamic measurements) since in this temperature region the measured signal is weak, meaning that all recombination processes are completed.

Considering Fig. 11, both the compatibilization and the clay introduction lead to a decrease in the area of the TL glow curve of iPP-based materials. To the contrary, for the sPP matrix, the area of the TL peak increases.

The difference in area is linked to the density of recombination event, to the probability a recombination event produces an excited state, and to the probability this excited state relax radiatively. The last two features are directly dependent on the nature of the recombination centre(s). Since positive ions are included with the clay we can suppose that the recombination process involves electrons and cations. Moreover, it has been shown in RIL measurements that it is probably oxidized PP and not ODA that is emitting. The decrease of the peak area observed in iPP matrix when nanoclay is introduced suggests a decrease in the number of trapped carriers. In sPP matrix it seems that there is an increase in the number of trapped carriers and/or number of radiative centres. This can be linked to an increase of the oxidation degree, particularly as regards $\mathrm{sP} 20 \%$. It seems that these variations are linked to the clay concentration rather than to the matrix nature since $\mathrm{iPP} 6 \%$ and $\mathrm{SPP} 6 \%$ have the same TS area.

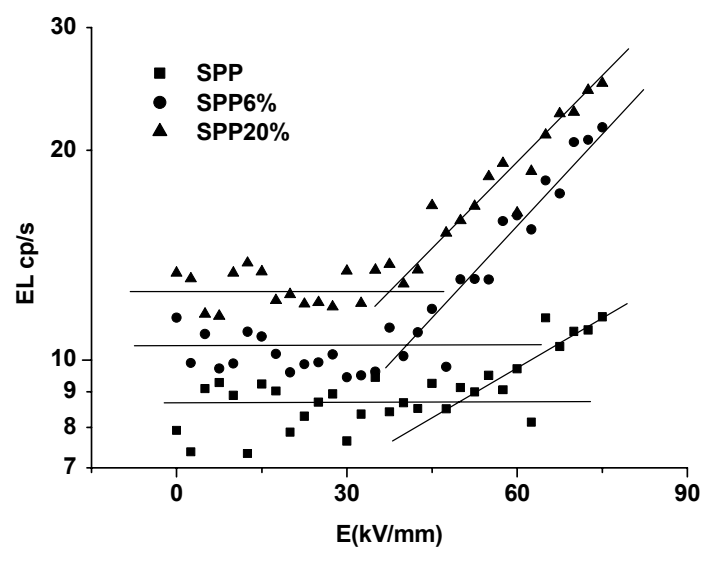

Fig. 12 EL (in counts per second) vs. field characteristic for sPP-based samples

Table 3. Threshold fields (in $\mathrm{kV} / \mathrm{mm}$ ) derived as EL inception field, $\mathrm{E}_{\mathrm{EL}}$ (cf. Fig. 12)

\begin{tabular}{lc} 
Sample & EEL \\
iPP & 55 \\
iPP20AR & 42 \\
iPP6\% & 47 \\
sPP & 50 \\
sPP6\% & 40 \\
sPP20\% & 37 \\
\hline
\end{tabular}

\section{Electroluminescence}

Figure 12 shows examples of EL vs. field characteristics obtained on sPP-based samples. The noise level was shifted vertically in order for the curves to be distinguishable. The EL onset field $-\mathrm{E}_{\mathrm{EL}^{-}}$(reported in Table 3) has been defined at the intersection between the lines represented in Fig. 12. In case of iPP-based samples, the trend is towards a decrease of the EL threshold with MA grafting and clay introduction. In sPP-based samples, filler introduction also decreases the EL threshold. The EL threshold decrease can be associated with the conductivity increase linked to the filler concentration ${ }^{(20)}$.

\section{Conclusion}

We have performed a broad optical caracterisation of PP/clay $\mathrm{NC}$ with a special attention to matrix nature (tacticity, blended or not) and clay concentration. The main results can be summarized as follows:

(1) It appears that compatibilisation of PP chain modifies strongly photoluminescence and absorption characteristics. The origin of these modifications are due to MA compatibilisation by products or/and to PP oxidized groups.

(2) In NCs, the optical characteristics are those of oxidized PP. It is probably due to the reduction of antioxidant efficiency.

(3) Light scattering in NCs is both clay and crystallinity dependent, the latter being the most influential factor.

(4) RIL shows that no additional radiative recombination centres are introduced upon MA grafting or filler introduction. It seems that oxidized PP is providing the recombination centres.

(5) The thermoluminescence curves show an increase in the number of trapped carriers in SPP matrix based NC and a decrease has been observed in iPP matrix based NC. These observations are in agreement with space charge density measured at $30-40 \mathrm{kV} / \mathrm{mm}$ 
after a poling period of 10000 s with by the pulse electro-acoustic method $^{(21)}$.

(6) Filler introduction in the matrix decreases the EL threshold.

Thermostimuled current measurements will be performed soon to complete the traps characterisation.

Overall, this work show that chemical/morphological modications needed for $\mathrm{NC}$ processing are critical for optical and luminescence properties. This could also be the case for electrical properties: it is highly challenging to relate changes of electrical properties with the nanometric nature of the clay.

\section{Acknowledgements}

We are grateful to the Freiburger Materialforschungszentrum and Institut für Makromolekulare Chemie, Albert-Ludwig University, Freiburg, Germany for providing samples.

(Manuscript received Feb. 23, 2006)

\section{References}

(1) T. J. Lewis : "Interfaces are dominant feature of dielectrics at the nanometric level": IEEE Trans. Dielectr. Electr.Insul., Vol.11, p.5 (2004)

(2) P. Reichert, H. Nitz, S. Klinkle, R. Brandsch, R. Thomann, and R. Mülhaupt : "Polypropylene/organoclay nanocomposite formation: Influence of compatibilizer functionality and organoclay", Macromol. Materials Engg., Vol.275, pp.8-17 (2000)

(3) G. Gorassi, M. Tortora, V. Vittoria, D. Kaempfer, and R. Mülhaupt : "Transport properties of organic vapors in nanocomposites of organophilic layered silicate and syndiotactic polypropylene", Polymer, Vol.44, pp.3679-3685 (2003)

(4) H. Qin, S. Zhang, C Zhao, G Hu, and M. Yang : "Flame retardant mechanism of polypropylene/organoclay nanocomposites based on polypropylene", Polymer, Vol.46, pp.8386-8395 (2005)

(5) T. Tanaka : "Dielectric nanocomposites with insulating properties", IEEE Trans. Dielect. Elect. Insul., Vol.12, pp.5 (2005)

(6) M. Kato, A. Usiki, and A. Okada : "Synthesis of polypropylene oligomer-clay intercalation compounds", J. Appl. Polym. Sci., Vol.66, pp.1791-1795 (1997)

(7) C. Zilg, D. Kaempfer, R. Thomann, R. Mülhaupt, and G. C. Montanari : "Electrical properties of polymer nanocomposites based upon organophilic layered silicates", IEEE CEIDP, pp.546-49 (2003)

(8) G. Teyssedre, L. Cisse, C. Laurent, F. Massines, and P. Tiemblo : "Spectral analysis of optical emission due to isothermal charge recombination in polyolefins": IEEE Trans. Dielect. Elect. Insul., Vol.5, pp.527-535 (1998)

(9) P. P. L. Jacques and R. C. Poller : "Fluorescence of polyolefins-2.Use of model compounds to identify fluorescent species in thermally degraded polymers", Euro. Polymer J., Vol.29, pp.83-89 (1993)

(10) S. Morlat-Therias, B. Mailhot, D. Gonzales, and J. L. Gardette : "Photooxidation of Polypropylene/montmorillonite nanocomposites. 1. Influence of nanoclay and compatibilizing agent", Chem. Mater, Vol.16, pp.377-383 (2004)

(11) S. Morlat-Therias, B. Mailhot, D. Gonzales, and J. L. Gardette : "Photooxidation of Polypropylene/montmorillonite nanocomposites. 2. Interactions with antioxidants", Chem. Mater, Vol.17, pp.1072-1078 (2005)

(12) G. Bogoeva-Gaceva, B. Mangovska, and E. Mäder : "Crystallization kinetics of maleic anhydride-modified iPP studied by POM", J. Appl. Pol. Sci., Vol.77, pp.3107-3118 (2000)

(13) Optical properties of polymers, G. H. Meeten, Ed., Elsevier Applied Science (1986)

(14) W. R. Krigbaum and I. Uematsu : "Heat and entropy of fusion of isotactic PP”, J. Polymer Sci. Part A, Vol.3, pp.767-776 (1965)

(15) J. C. M. Suarez, M. F. V. Marques, D. E. B. Lopes, and J. D. Rego : "Fracture study of syndiotactic and isotactic PP", Key Engineering Materials, Vol.230-232, pp.255-258 (2002)

(16) J. Ma, S. Zhang, Z. Qi, G. Li, and Y. Hu : "Crystallization behaviors of polypropylene/montmorillonite nanocomposites", J. Appl. Pol. Sci., Vol.83, pp.1978-1985 (2002)

(17) F. Perrin-Sarazin, M. T. Ton-That, M. N. Bureau, and J. Denault : "Microand nano-structure in polypropylene/clay nanocomposites", Polymer, Vol.46, pp.11624-11634 (2005)
(18) F. Massines, P. Tiemblo, G. Teyssedre, and C. Laurent : "On the nature of the luminescence emitted by a polypropylene film after interaction with a cold plasma at low temperature", J. Appl. Phys., Vol.81, pp.937-943 (1997)

(19) R. J. Fleming and J. Hagekyriakou : "Thermoluminescence in polymers", Radiation Protection Dosimetry, Vol.8, pp.99-116 (1984)

(20) M. Ambid, D. Mary, G. Teyssedre, C. Laurent, and G. C. Montanari : "Investigation of electrical and luminescent properties of polypropylene-based nanocomposite materials", Proc. IEEE-ISEIM, pp.227-230 (2005)

(21) G. C Montanari, D. Fabiani, F. Pamieri, D. Kaempfer, R. Thomann, and R. Mülhaupt : "Modification of electrical properties and performance of EVA and PP insulation through nanostructure by organophilic silicates", IEEE Trans. Dielect. Elect. Insul., Vol.11, pp.754-760 (2004)

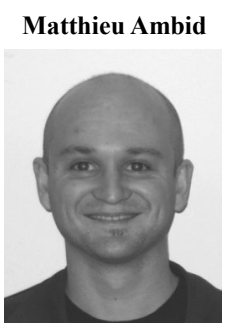

(Non-member) was born in 1979 in Nîmes, France. He received his Engineer Degree in physics in 2003 at the National Institute for Applied Science (INSA) of Toulouse and received master degree in "materials and technology for electronic device" the same year. Currently, he is preparing $\mathrm{PhD}$ degree in the solid dielectric and reliability department of the Electrical Engineering Laboratory in Toulouse. His research topic is the study of transport mechanisms in polypropylene/clay nanocomposites.

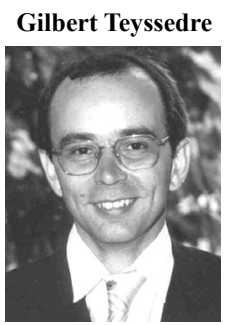

(Non-member) was born in 1966 in Rodez, France. He received his Engineer Degree in materials physics in 1989 at the National Institute for Applied Science (INSA) and graduated in solid state physics the same year. Then he joined the Solid State Physics Lab in Toulouse and obtained the Ph.D. degree in 1993 for a work on transition phenomena and electro-active properties of fluorinated ferroelectric polymers. He entered the CNRS in 1995 and has been working since then at the Electrical Engineering Lab in Toulouse. His research activities concern the development of luminescence techniques in insulating polymers with focus on chemical and physical structure, degradation phenomena, space charge and transport properties. He is currently leading a team working on the reliability of dielectrics in electrical equipment.

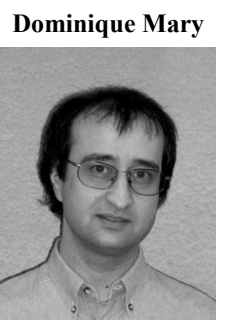

(Non-member) was born in 1967 in Chenôve (Bourgogne), France. He received his M.S. in physics (electronic material and component) in 1990 from the Paul Sabatier University of Toulouse, France and graduated in the same year. Then he joined the Electrical Engineering Laboratory in Toulouse (LGET). He also received the Ph.D. degree in 1994 for "a work on evidence and study of luminescence in insulating polymers under electric field or interacting with a cold plasma : Aging diagnostic and application". During his thesis (1994), he spent few months in Quebec (Canada) in the Research Center of Hydro Quebec. He joined the University in 1995 like associate professor and has been working since then at the LGET. His research activities concern the development of approach to electrical aging and space charge in polymeric materials based on electroluminescence analysis. $\mathrm{He}$ is Vice-President of Electrical and Electronical Society (Midi-Pyrenees group). 
Christian Laurent (Non-member) was born in Limoges, France, in 1953.

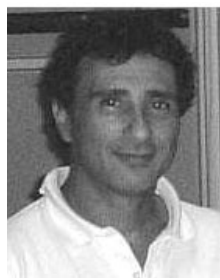

He studied solid state physics at the National Institute for Applied Sciences in Toulouse and received the Eng. degree in physics in 1976 dealing with experimental and modeling activity. He joined the Electrical Engineering Laboratory at Paul Sabatier University in 1977 to study electrical treeing and partial discharge phenomena, which were the topics of his Dr. Eng. Degree (1979). He joined CNRS (National Centre for Scientific Research) in 1981 and got his Doc-ès Sc. Phys. in 1984. In 1985, he spent one year as a post-doctoral fellow with the IBM Almaden Research Center, where he studied plasma-polymerized thin films. Back in Toulouse he developed an approach to electrical aging in polymeric materials based on luminescence analysis. He is now in charge transport and aging. He is currently Research Director at CNRS and Director of the Electrical Engineering Laboratory in Toulouse.
Gian Carlo Montanari (Non-member) was born on 8/11/55. In 1979, he

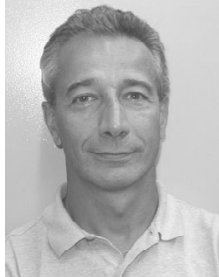
took the Master degree in Electrical Engineering at the University of Bologna. He is currently Full Professor of Electrical Technology at the Department of Electrical Engineering of the University of Bologna, and teaches the courses of Reliability and statistics for electrical systems and Innovative electrical technologies. He has worked since 1979 in the field of aging and endurance of solid insulating materials and systems, of diagnostics of electrical systems and innovative electrical materials (magnetics, electrets, superconductors). $\mathrm{He}$ has been also engaged in the fields of power quality and energy market, power electronics, reliability and statistics of electrical systems. He is IEEE Fellow and member of AEI and Institute of Physics. Since 1996 he is the Italian representative in CIGRE and President of the Italian Chapter of the IEEE DEIS. $\mathrm{He}$ is convener of the Statistics Committee and member of the Space Charge, Multifactor Stress and Meetings Committees of IEEE DEIS. He is Associate Editor of IEEE Transactions on Dielectrics and Electrical Insulation. He is founder and President of the spin-off TechImp, established on 1999. He is author or coauthor of about 380 scientific papers. (IEEE member since 1987, senior member since 1990, fellow since 2000 ) 